\title{
Versuch einer Erklärung von Psalm r8.
}

Von Dr. Hans H. Spoer-in Jerusalem.

In der Auslegung der Psalmen ist man heute noch im allgemeinen zu sehr von der Ansicht beeinflußt, daß der Psalter das Choral- resp. Erbauungsbuch der jüdischen Gemeinde war. Wenn dieses auch wohl in späterer Zeit der Fall war, so waren diese Lieder doch wohl nicht alle in der Absicht gedichtet, um beim Gottesdienste oder zur privaten Erbauung benutzt zu werden. Viele der Psalmen verraten ihren religiösen Charakter ohne weiteres, in andere ist er aber erst durch spätere Auslegung hinein gelegt worden; vielleicht ist auch manchem alten Heldenliede ein messianischer Charakter untergeschoben worden in einer Zeit, wo der Glanz des nationalen Heldentums für immer versunken war und der Wunsch nach einem Helden-Messias sich, in Erinnerung an die Verheißungen Gottes, natürlicherweise stärker regte als jemals. Ein solches Vorgehen, von welchem selbst manche unserer modernen Gelehrten nicht ganz frei sind, erinnert mich an eine Begebenheit, die sich im Hause des englischen Dichters Browning zutrug. Die Londoner "Browning-Society“, die die vornehmsten literarischen Geister als Mitglieder zählt, hat es sich zur Aufgabe gemacht, die Werke dieses Dichters zu interpretieren. Browning, der übrigens oft sehr belustigt war über die „tiefen“ Gedanken, die in manche seiner Gedichte hinein gelegt wurden, bat eine mir bekannte Dame, sie möchte ihm doch mitteilen, wie man sein Gedicht "Childe Roland" dort ausgelegt habe. Er multe dann zu seinem gröbten Gaudium hören, daß man in dem Gedichte ein psychologisches Problem des Lebens dargestellt fand, während 
Browning selbst nur die Wanderungen und Abenteuer eines mittelalterlichen Reisenden beschrieben hatte!

Ohne Zweifel besitzen wir in der Psalmsammlung aufer Liedern religiösen Inhalts, die der Gemeinde- oder Privaterbauung dienten, eine grofe Anzahl Gedichte, die dem alltäglichen Leben der Juden entsprossen sind und infolge der Umstände, denen sie ihr Dasein verdanken, eine mehr oder minder tief religiöse Stimmung verraten. Ich brauche nur auf die deutschen Freiheitslieder hinzuweisen als Parallelen für das eben Gesagte; man denke nur an Körners ergreifendes Gedicht „Gebet während der Schlacht“. $\mathrm{Daß}$ derartige Lieder der Psalmsammlung einverleibt werden konnten, ohne Anstoß zu erregen, ist selbstverständlich. Es widersprach dem religiösen Gefühle der Israeliten, wie noch heute den palästinensischen und ägyptischen Fellahen, nicht, selbst in ihren gewöhnlichen Liedern der Mitwirkung Gottes in ihrem Tun und Handeln Ausdruck zu geben, und es ergibt sich daher diese Mischung des Religiösen mit dem Nichtreligiösen, welcher Art das Letztere auch immer sei. Wir müssen solche Lieder strenge scheiden von den religiös erbauenden Liedern.

$\mathrm{Da}$ das psalmodierende Ich in manchen Fällen auf die Gemeinde hinweist, muß anstandslos zugestanden werden, wenn die Gedichte ihrem Inhalte nach sich als Gemeindegesänge erweisen. In vielen Fällen aber gibt der Dichter seinem subjektiven Empfinden Ausdruck, ohne sich als Typus anderer hinzustellen, die sich, vielleicht ohne sein Wissen, in derselben Lage befinden. Dab solche in dem Dichter ihren Wortführer und in seinem Gedichte eine Schilderung ihrer eigenen Leiden erkennen, bleibt natürlich nicht aus; denn in dem Grade wie der Verfasser Dichter ist, muß er den Empfindungen und Regungen des Menschengeschlechtes Ausdruck geben, bewußt oder unbewußt. In dem Dichter des geistlichen Liedes kann man ohne weiteres den Sprecher seiner Glaubensgenossen erkennen; dieses ist jedoch nicht der Fall in dem Volksliede, welches ganz andere Zwecke verfolgt und nur mit Zwang eine Erklärung zuläßt, die sich auf die Allgemeinheit bezieht. Aus diesem Grunde kann man keine allgemein gültige 
Regel für die Bestimmung des "Ich" in den Psalmen aufstellen, bis eine vollständige Scheidung zwischen weltlichen- oder Volksliedern und religiösen (vielleicht gar Gemeinde-)Psalmen gelungen ist.

In Ps. 18, der, wie ich unten weiter ausführe, aus zwei Gedichten und einem Fragmente zusammengesetzt scheint, findet man beides, das "Ich" der Gemeinde und das "Ich" des Individuums in dem Siegesliede Ps. B.

Es ist schon vielfach darauf hingewiesen worden, daß Ps. 18 in seinem ganzen Umfange nicht davidischen Ursprunges sein kann. Da jedoch von manchen Gelehrten ${ }^{\text {x }}$ der Psalm wenigstens teilweise David zugeschrieben wird, oder gar ganz, wenn auch in Überarbeitung vorliegend, so dürfte es vielleicht von Interesse sein, die Argumente, die gegen eine davidische Autorschaft dieses Psalmes sprechen, zusammenzustellen.

1) Die Bezugnahme auf den Tempel in v. 7 .

2) עם עני עי 28 ist technische Bezeichnung der gesetzestreuen Israeliten v. $22 \mathrm{f}$, die in der späteren Zeit schwere Verfolgungen erlitten.

3) Der weite politische wie religiöse Ausblick, der den Versen 32, 44 und 50 eigen ist.

4) Die Bezugnahmen auf das Deuteronomium, besonders auf das Kap. 32, sind zahlreich:

Ps. 18 v. 3. 31 vgl. mit Dt. 33, 29; v. 8.9 vgl. mit Dt. 32, 22; v. 19 יום אידם vit. 19 . 32,35 ; v. 24 a vgl. mit Dt. I8, I3; v. 27 פתלתל Dt 32 תgl. mit 5 ; v. 27 b vgl. mit Dt. 32, 5b; v. 32 הלוm vgl. Dt. 32, 15. 17, außerdem vom Gott Israels nur in späten Stellen, z. B. Hiob $42 \mathrm{Mal}$; Ps. 50, 22; I I4, 7; 139, 19; Prov. 30, 5; Jes. 44, 8; Hab. 3, 3; Neh. 9, I7 zitiert Ex. 34,3 , wo ל steht ${ }^{2}$; v. $34 \mathrm{~b} \cdot \mathrm{vgl}$. mit Dt. 32 , 13a; v. 37 vgl. mit Dt. 32, 35; v. 39 קום im selben Sinne gebraucht wie in Dt. 33, I I ; v. 39 a vgl. mit Dt. 33, I I c-d; v. 45 b vgl. mit Dt. 33, 29 e.

I Graetz (Commentar z. d. Psalmen I S. 207) hält die zweite Hälfte des Psalmes für davidisch. Briggs (Messianic Prophecy S. 143) sagt: „It is probably Davidic in origin, as it seems to reflect his historic experience". Baethgen (Handkommentar S. 49) vermutet in dem Psalme ein davidisches Siegeslied.

2 Hebrew Lexicon based on the Lexicon of Gesenius by F. Brown. 
5) Die Bezeichnung צור für Jahwe Ps. 18, 3. 32. 47 findet sich nur in der späteren Literatur, besonders in den Pss. und Dt. 32.

Ps. 19,$15 ; 28,1 ; 31,3 ; 62,3.7 .8 ; 71,3 ; 73,26 ; 75,6$ (1. IXXX et al.); 78, 35;89, 27; 92, 16; 94, 22; 95, 1; 144, 1; Dt. 32,4 . 15. 18. 30. 31. 37 ; Jes. 17 , 10; 26,$4 ; 30,29 ; 44,8$; II S. 23, 3; Hab. I, I2 und in den Parallelstellen zu Ps. 18 in II S. 22.

צו findet sich auch in einigen Eigennamen in Stellen, die P angehören. אליצור Num. 1, 5; 2, 10; 7, 30.35; 10, 18; צורשרי Num. I, 6; 2, 12; 7, 36.41; 10, 19; פרהצור Num. 1, 10; 2, 20; 7, 54. 59; 10, 23; צוריאל Num. 3, 35 .

6) Das Kapitel Dt. 32, mit welchem Ps. 18 so viele Berührungspunkte hat, gehört vielleicht gegen das Ende des Exiles', jedenfalls nicht in vorexilische Zeit ${ }^{2}$.

7) Aramaismen, späte, sowohl wie seltene Wörter sind ziemlich zahlreich.

v. 2 רח im Sinne von lieben ist aramäisch; siehe jedoch die Emendationen.

v. 3 Oֵלק mein Fels nur in den Pss. auf Jahwe angewandt II S. 22, 2; Ps. 3I, 4; 42, 10; 7I, 3 .

v. II דהה schweben nur hier II S. 22; Dt. 28, 49; Jer. 48,$40 ; 49,22$.

v. I5 רבב schießen nur hier IIS. 22; Gen. 49, 23.

v. I7 משת ziehen nur hier IIS. 22; Ex. 2, 10 im Perf. Qal.

v. 19 Unglück hauptsächlich in der Weisheitsliteratur und späteren Schriften: Jer. 18, 17; 46, 21; 48, 16; 49, 8.32; Hes. 35, 5; Hiob 18, 12; 21, 17; 30, 12; 31, 3. 23; Prov. I, 26. 27; 6,$15 ; 17,5 ; 27,10 ;$ Ob. v. 13; Dt. 32, 35; II S. 22.

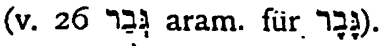

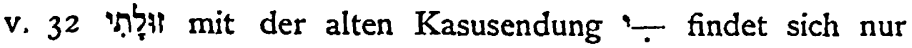
hier und in Dt. I, 36; 4, 12; Jos. I1, 13; I Kg. 3, 18; 12, 20.

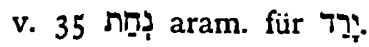

v. 46 חרוt zittern einzige Stelle.

I Stcuernagel, Handkommentar.

2 Driver, International Crit. Commentary. 
8) Siehe auch kritische Anmerkungen.

Abgesehen vom Inhalte, verbieten die sprachlichen Eigentümlichkeiten des Psalmes, daß wir den Ursprung des Gedichtes doch wenigstens noch in die Königszeit verlegen dürfen. Die vielen Berührungspunkte mit anderen literarischen Erzeugnissen weisen uns vielmehr in eine nachexilische Zeit. Die genaue Abfassungszeit des Psalmes kann jedoch nur bestimmt werden durch die historischen Hinweise, die im Liede selbst enthalten sind. Eng verknüpft mit der Frage nach der Abfassungszeit ist die Frage nach der Einheit des Psalmes, deren Beantwortung die Antwort auf die erstere Frage in sich einschließt.

Duhm sieht in der Wortfülle, die alle Ausdrücke und Bilder für den Begriff Schutz zusammenhäuft, einen Beweis, daß der Dichter beabsichtigte ein langes Lied zu verfassen. Dem gegenüber scheint mir jedoch gerade diese Wortfülle als ein Beweis, daß der Verfasser beabsichtigte in möglichst gedrängter Form ein lebendiges Bild von dem ihn schützenden Jahwe zu entwerfen. Hätte der Verfasser die Absicht gehabt; ein längeres Lied zu schreiben, so wäre diese nur einen Gedanken ausdrückende Wortfulle ein Fehler, da der Rest des Gedichtes im Vergleiche zum ersten Verse viel zu mager ist; wie ein brausender Strom setzt das Lied ein, um als ein verhältnismäßig armseliges Rinnsal auszulaufen. Bei einem kürzeren Gedichte ist jedoch ein solches Beginnen sehr wirkungsvoll, da das Majestätische seines Anfangs bis zum Schlusse kraftvoll durchdringt.

Graetz hat schon die Existenz zweier Lieder, die zu Ps. 18 verschmolzen sind, erkannt I) v. I-29; 2) v. $33-50$, v. 5 I ist liturgisches Anhängsel. Jedoch ist meines Wissens noch von niemand die eigentümliche. Stellung der Theophanie v. 8-16 gebührend hervorgehoben worden. Die Pss. bieten hierfür keine Parallelen; denn Ps. 144, I-11 ist von diesem Psalme abhängig. Die Theophanie hängt nur lose mit den übrigen Teilen des Psalmes zusammen, und man fühlt instinktiv, daß eine solche großartige Manifestation göttlicher Kraft in keinem Verhältnisse steht $z u$ der Gefahr, in welcher sich der Mann befindet. Vers 8 setzt ganz 
abrupt ein nach v. 7, erst in v. 14 wird der Gott beim Namen genannt. Stände v. 14 nach v. 7, so hätte man Jahwes Antwort auf den Hülferuf seines Dieners; so aber folgt zuerst eine grobartige Offenbarung der Macht Jahwes in der Natur, dann läbt er seine Stimme erschallen, hierauf folgt wiederum eine Offenbarung der Macht Jahwes und dann endlich die Rettung des Hartbedrängten. Natürlicher ist der direkte Anschluß von v. 17 an v. 7 , vielleicht mit Einschub von v. 14 hinter v. 7 und mit Ausschlub von v. $8-13+15-16$, so dab sich folgende natürliche Dreiteilung ergäbe: Hülfegeschrei, Antwort und Rettung. Es findet sich keine Bezugnahme in der Úberschrift auf die Theophanie. Aus diesen Gründen betrachte ich die Theophanie als ein selbständiges Stuick, welches vom letzten Redaktor in den Psalm hineingeflickt wurde, siehe S. 155. Das Gedicht ist nicht vollständig und schliebt nur die Verse 8-13. 15-16 ein; v. 17-18, die Gunkel ${ }^{2}$ hinzuzieht, gehören nicht mehr zur Theophanie, denn v. I 7 schließt sich direkt an v. 7 resp. v. 14 an. Jahwe ist in seinem Tempel v. 7 und muß sich hinunter beugen, um dem bedrängten Sänger zu helfen v. 17. „Wasser": v. 17 ist hier bildlich gebraucht und ist parallel zu „Wogen des Todes", „Bäche des Totenreiches" in v. 5 .

Das Stück v. 8-13+15-16 bildet eine gute Parallele zu dem Kampfe zwischen Marduk und Tiâmat, nur fehlt die Beschreibung des eigentlichen Kampfes, diese ist jedoch ersetzt durch die zweite Hälfte des Psalmes. Ein kurzer Hinweis auf die Ähnlichkeit dieses Stückes mit dem Schöpfungsepos der Babylonier möge dazu dienen, das, was im Zusammenhange mit der Theophanie gesagt wurde, zu bekräftigen.

Wie nach Ps. 18, 8 die Erde schwankte und die Grundfesten der Berge erbebten, als Jahwe ergrimmte, so „schrie auf Tiâmat ungestüm sich aufbäumend, in Tiefsten durch und durch erbebte ihr Gebein"'. Wie Jahwe auf dem Cherub fuhr, so fuhr Marduk auf dem „unvergleichlichen Wagen". Die Bewaffnung beider Gottheiten ist die gleiche: Bogen, Pfeile und Blitze. Marduk schuf

× Schöpfung und Chaos S. ro3.

2 Zimmern in "Schöpfung und Cha.os" S. 4r2. 
einen Orkan zu seinem Beistande, während Jahwes Odem dem Feinde zusetzt. Tiâmat selbst ist beibehalten worden, obgleich nicht unter ihrem biblischen Namen תהום, sondern in den Umschreibungen v. אפיקי ימים ומוסדי הרים , הארץ (Duhm) v. ומים 8. ימים entspricht natürlich dem Apsu.

Diese Berührung mit dem babylonischen Mythos tritt stark hervor in den jüdisch-apokalyptischen Schriften. Ein himmlisches Wesen, z. B. der Erzengel Michael, streitet für das jüdische Volk, Dan. 12, I. Jedoch ist es, wie Bousset ${ }^{x}$ ausführt, meistens die Gottheit selbst, die diesen Kampf unternimmt, also genau wie in den vv. 8-I3+I5-I6 von Ps. I8, wodurch der Ps., falls er als Einheit betrachtet wird, in diese Zeit gerückt werden muß. Es macht sich jedoch in den folgenden Versen ein Widerspruch geltend, da gemä $\emptyset$ vv. 30.32 bis zum Schluß der "Ich" des Psalmes den Kampf führt. Wir haben demnach eine Verschmelzung zweier Ideen, erstens „die Niederwerfung und Vernichtung der feindlichen Weltmächte durch Jahwe" und zweitens den Kampf eines israelitischen Helden mit seinen Feinden. $\mathrm{Da}$ das eine Motiv das andere ausschließt, so muß angenommen werden, daß hier zwei selbständige Dichtungen zusammengeflossen sind. Das Stück v. $8-13+15-16$ erweist sich demnach als selbständiges Stuick. Die Frage nach dem $Z$ wecke dieser Einschaltung von v. 8-I3+I5-I6 wird in Verbindung mit v. $5 \mathrm{I}$ erörtert werden.

Nach Ausscheidung dieses Fragmentes bleiben noch v. $\mathbf{I}-7+$ I4+17-5r. Aus unten weiter ausgeführten Gründen schlage ich folgende Zweiteilung vor: v. $1-7+14+17-29+31=P s$. A; v. $3 \dot{0}+32-5 \mathrm{I}=\dot{\text { Ps. }} \mathrm{B}$.

Ein Vergleich des Inhaltes beider Lieder legt sofort die Grundverschiedenheit ihrer Hauptgedanken dar.

Die Situation des Sprechers in. Ps. A ist ganz verschieden von derjenigen des "Ich" in Ps. B. Der psalmodierende Ich in Ps. A -ist unterdrückt und erleidet wegen seiner Gerechtigkeit und um seiner religiösen Überzeugung .willen Verfolgung. $\mathrm{Er}$ weiß

I Religion des Judentums S. $207 \mathrm{f}$. 
sich jedoch, wegen seiner treuen Anhänglichkeit an das Gesetz, als speziell begünstigt von Jahwe. Infolgedessen fühlt er sich trotz aller Bedrückung so sicher, da $\mathrm{b}$ er seine Gedanken in jene mystische messianische Zukunft projizieren kann, in eine Zeit hinein, in der der gesetzestreue Israelit über den renegaten Juden und Heiden triumphieren soll, v. 28. In diesen frommen Meditationen findet er Vergessenheit für sein gegenwärtiges Leiden.

Die Gottheit, der er sich ergeben hat, ist ein sittliches Wesen; denn das Verhältnis zwischen ihr und dem Sänger ist auf Gerechtigkeit aufgebaut, v. 25. Wenn er sich auch in besonderem Schutze seines Gottes weib, so hängt doch Jahwes Gunst ganz und gar von der Reinheit seiner Handlungen ab, v. $27 \mathrm{f}$. Trotz seiner scheinbaren Selbstgerechtigkeit, v. 22-24, spricht aus den Worten des Dichters eine wahre Frömmigkeit; er weist es von sich, daß er seine Erfolge seinem eignen Können und Wissen verdanke, sondern in Demut schreibt er sie Gott zu, der seine Leuchte scheinen läßt und sein Dunkel helle macht, v. 29. - Es ist der sittliche Charakter seines Gottes, der den Dichter von Anfang bis zum Ende mit Vertrauen und Hoffnung auf einen guten Ausgang dieses ungleichen Kampfes erfüllt. Dieser Kampf ist nicht ein physisches Ringen, weder Beschreibungen von blutigen Szenen noch Schlachtengetöse dringen störend in das religiöse Bekenntnis des psalmodierenden "Ichs". Ein Hauch des tiefsten Friedens zieht durch das ganze Gedicht: Es ist ein geistlicher Hymnus, welchen die Gemeinde zum Lobe Jahwes erschallen läbt, auf dessen Gerechtigkeit bauend, sie sich eines glücklichen und friedlichen Ausganges aller Drangsal gewi $\lesssim$ ist. Nicht durch Macht noch durch Gewalt sondern durch den Geist Jahwes der Heerscharen wird der Sieg errungen.

Wenn ich Duhms Ausführung recht verstehe, so ist es gerade der in v. 25. 29. $3 \mathrm{I}$ enthaltene ethische Gedanke, der ihn mit Mißtrauen gegen ihre Echtheit erfullt, und auf Grund hievon stellt er den Verfasser als mittelmäßigen Schriftsteller hin. Aber damit ist der Sache nicht abgeholfen. In ihrem gegenwärtigen Zusammenhange nehmen sich diese Verse seltsam genug aus, füllen aber 
genau ihre Stelle aus, wenn die hier vorgeschlagene Scheidung vorgenommen wird.

Wie ganz anders ist jedoch der Geist, der aus dem zweiten Liede spricht. Der "Ich" des Psalmes hat es auf sich genommen, mit dem Schwerte das Unrecht zu rächen, welches seine Feinde ihm angetan haben, v. $34 \mathrm{f}$; jedoch erfreut er sich hierbei des Beistandes des alten Kriegsgottes Israels, dessen Charakter als solcher in jeder Zeile scharf zum Ausdruck kommt. Die Schrecken eines orientalischen Krieges sind ungeschminkt dargestellt und wie es scheint mit einer gewissen Genugtuung seitens des Dichters. Kein Wort des Friedens unterbricht die blutigen Szenen. Der Aufruhr des Schlachtens ist nur dann und wann übertönt von dem Schrei eines der so grausam Behandelten um Hülfe von Jahwe; aber dieser Gott ist nicht „ein Schild für Alle, die sich bei ihm bergen", wie der Dichter des Ps. A mit prophetischer Stimme verkündete. Der herzzerreißende Ruf um Hülfe des tödlich Getroffenen erweckt kein Mitleid weder in der Brust des Kriegers noch in seinem Gotte, beiden fehlt jede Spur von Großmut, wenn nicht jedes Gefühl der Menschlichkeit. Der Gott, mit dem der gefeierte Krieger seinen Rachekampf gegen seine Feinde ausführt, ist kein sittliches Wesen, sein Charakter ist der eines wilden Kriegsgottes.

Wir erkennen selbst nach dieser kurzen Darlegung, daß in diesen beiden Gedichten zwei einander vollständig fremde Gedanken zum Ausdruck gebracht sind, selbst wenn Ps. B hyperbolisch verstanden sein wollte.

Die Gottheit in Ps. A ist ein sittliches Wesen. Der Kampf ist ein unblutiger und in dem Kampfe bewährt sich zuletzt die Überlegenheit derer, die das Gesetz Jahwes befolgen, über diejenigen, welche es verachten.

Die Gottheit in Ps. B ist der alte israelitische Kriegsgott, und der Kampf ist ein blutiger Rachekrieg, v. 48. -

I Spoer, The Origin and Interpretation of the Telragrammaton in "The American Journal of Semitic Languages" 1901. S. 31. 
Ps. A bietet weiter keine historischen Anhaltspunkte, mit Hülfe welcher man die Abfassungszeit des Liedes näher bestimmen könnte; anders liegt die Sache mit Ps. B. Die inneren Zeugnisse dieses Liedes weisen alle auf die Zeit des Johannes Hyrkanus 135-104 v. Chr. hin und es ist dieser gewandte Staatsmann und unerschrockene Krieger, der in diesem Liede gefeiert ist. Hyskanus nennt sich auf seinen Münzen ראש חבר היהודים, ein Ausdruck, dem derjenige in v. 44 ähnlich ist, obgleich hier der Dichter, vielleicht ein Höfling, in seinem Lobe etwas zu weit gegangen ist. Verse 30 und 32 nehmen vielleicht Bezug auf Hyrkanus' Einnahme von Samaria ', Madeba ${ }^{2}$, Adora und Marissa. Die inneren Feinde v. 42, gegen welche sich der Held wehren mußte, sind die pharisäische Partei, mit welcher es zu einem offenen Bruche kam, als Hyrkanus die pharisäischen Satzungen abschafite3. Mit Recht weist Duhm darauf hin, daß der Gebrauch von in Verbindung mit den Feinden, den Schluß notwendig macht, daß die Kämpfe zwischen Juden und Juden (mindestens Samari-

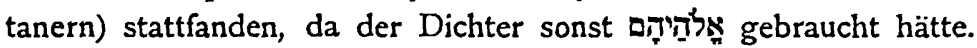
Solcher Kämpfe gab es viele unter Hyrkanus. Die Aufstände ['] ריבֵ (so nach II S. 22 und Codex U zu Ps. I8) lassen sich auch am besten aus dieser Zeit heraus erklären4. Vor seinem Tode gelang es Hyrkanus viele der kleinen Nachbarstaaten und Städte in seine Gewalt zu bringen, so daß er wohl in den Augen eines nationalistischen Dichters als "Haupt von Nationen" v. 44. 48 beschrieben werden mochte. Der "Mann der Gewalttat" ist kein anderer als Antiochus VII. Sidetes, durch dessen plötzlichen Tod im Jahre 129 v. Chr. der Druck von Hyrkanus genommen wurde, welcher jetzt seinem Ehrgeize keinen $Z_{\text {wang }}$ mehr aufzuerlegen brauchte, und dieses drückt der Dichter aus in den Worten „Der Gott .... der ... meinen Weg ohne Anstoß sein läßt“ v. 33. - Der Ausbruch unbändiger Grausamkeit in v. 43, mit welcher

I Josephus, Antt. XIII I0, 2-3.

2 ib. Antt. XIII I, 2; 15, 4; XIV I, 4.

3 ib. Antt. XIII 10, 5 .

4 ib. Bell. Jud. I 2, 8. 
der Held seine Feinde vernichten will, läbt sich leicht erklären aus der Bedrückung des Hyrkanus durch Antiochus VII., den Zerstörer der durch Simon errungenen Freiheit; jetzt wo Sidetes nicht mehr ist, kann Hyrkanus seinem Rachegefühl freien Lauf lassen und die Schmach, die das Schleifen der Mauern ${ }^{x}$ Jerusalems auf ihn gebracht hatte, im Blute seiner Feinde abwaschen. Es ist weiter bemerkenswert, daß in keiner Weise des Gesetzes Erwähnung geschieht. Der Held des Gedichtes ist ein Diener Jahwes; seine Religion ist frei von allen pharisäischen Anhängseln und hierin wieder berührt sich das Gedicht mit dem, was wir von Hyrkanus in betreff seiner Stellung zum strengen Judentum wissen.

Der geschichtliche Inhalt von Ps. B weist also auf eine Abfassungszeit nach $129 \mathrm{v}$. Chr.

Zum Schlusse möchte ich noch die Frage aufwerfen: welches war wohl der Grund für die Verschmelzung dieser Gedichte? Die Geschichte der Makkabäer scheint mir die Frage zu beantworten. Das starke makkabäische Fürstenhaus wurde von den „Frommen“ als ein messianisches Geschlecht angesehen ${ }^{2}$. Das populäre Siegeslied vielleicht eines Höflings, auf Hyrkanus' Taten gedichtet, drückte in mancher Hinsicht sehr wohl die messianischen Erwartungen der Zeit aus und eignete sich deshalb sehr gut als Hymnus auf den makkabäischen Messias, aus welchem Grunde die Beziehung auf den Messias in v. 5 I (siehe krit. Anm.) später dem Gedichte hinzugefügt wurde (siehe S. 159). Als jedoch später die Makkabäer von den Frommen als Usurpatoren angesehen wurden, hat man dieses messianisch-makkabäische Lied mit einem anderen Liede, Ps. B, welches dem Kreise der Frommen entstammte, verbunden, um auf diese Art das Gedicht allgemein messianisch zu gestalten (siehe krit. Anm. zu v. 50 f.). Der messianischen Erwartung der Zeit folgend, kam dann auch noch schlieblich das theophaniscke Fragment hinzu.

I Josephus Ant. XIII 8, 2-3.

2 Bousset op. cit. S. 210. 


\section{Textkritische Anmerkungen.}

v. 2 fehlt in II S. 22, wird von Duhm gestrichen, scheint mir

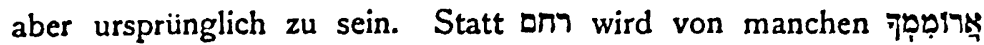
gelesen, so Prof. D. Stade, wie er die Güte hatte, mir mitzuteilen.

v. 3. Das Metrum dieses Verses ist ganz aus der Ordnung, durch einfache Einsetzung des Schlusses von II S. 22, 3 (Baethgen, Duhm et al.) wird die Schwierigkeit nicht gehoben. Man streiche im ersten Stichos יהוה nach P. und lese analog "mein Fels und meine Burg" אלי ו ו אורי - Jahwe ist in den beiden ersten Stichen mit sturmfesten Bauwerken, im dritten jedoch mit Waffen, verglichen, d.h. Schild und Rettungshorn; hinternach folgt משנבי "meine Veste", womit wieder auf den ersten Vergleich zurückgegriffen wird, das Wort klappt nach, außerdem hat dieser Stichos vier Hebungen, während alle übrigen nur drei haben, man streiche

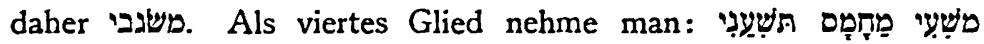
II S. 22, welches direkt an v. c anknüpft. מוּ II S. 22 streiche ich aus denselben Gründen wie משל Ps. 18. Wir gewinnen auf diese Art vier Stichen zu drei Hebungen. Man emendiere natür-

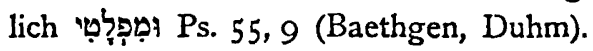

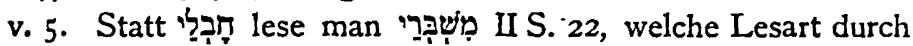
den Parallelismus bedingt wird (Baethgen, Duhm).

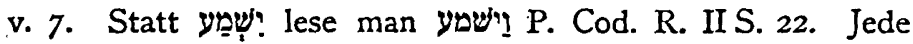
zweite, dritte etc. Zeile von Psalm $A$ ist mit der Konjunktion eingeleitet; wo sie fehlt, ist sie nach LXX, P. oder IIS. zu ergänzen: 2. B. 17b P., 18b, 19b, 20b P., 21 b LXX P., 22 b, 23b, 24b, 25 b LXX P., 26b LXX P., 27b, 28b, 29b, II S., 3I b P. Man streiche des Rhythmus halber לְ na nach II S. 22 (Baethgen).

v. 14. v. c ist' nach II S. 22, LXX $z$ u streichen.

v. I7. Man lese 'Iחקי LXX, P. und ebenso nach P. (v. I6) i cf. v. 17.

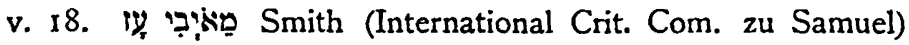
denkt die Schwierigkeit, die durch das Wort iv entstanden ist, durch Einschiebung eines $2 \mathrm{zu}$ heben. Vielleicht liest man besser 


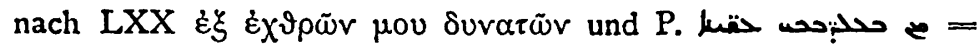

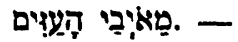

v. 19. Des Metrums halber schreibe man יל-.

v. 20. Die Lesart II S. 22 hat korrektes Metrum, $3+3$, während der Psalm nur $2+3$ Hebungen hat. Man lese יהלצy+? P. (s. v. 7).

v. 21. Man lese כבר LXX P. (s. v. 7) und des Rhythmus halber.

v. 22. Streiche Maqqēph des Rhythmus halber.

v. 25. Man lese כבר + LXX P. (s. v. 7). Das Metrum dieses

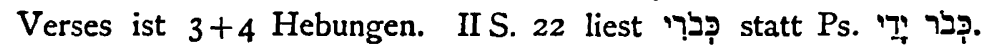
Des Metrums wegen ist die Lesart II S. vorzuziehen, da außerdem כברי seine Erklärung in v. 21 findet.

v. 26. נבר fehlt in P. Da es nicht in Verbindung mit und נבר v. 27 gebraucht ist, ist es wohl irrtümlich vor תמים geschrieben worden, vielleicht, wie Duhm annimmt, für das darunterstehende נבר va 27. Mit der Stichos eine Hebung zu viel. - Man lese $\Delta y+1$ LXX P. (s. v. 7) vv. 29. 31. Diese Verse beschreiben den sittlichen Charakter der Gottheit und sind von einander getrennt durch v. 30, welcher von $\mathrm{zu}$ erwartendem kriegerischem Erfolge des "Ich" spricht. Nach Duhm wird v. 30 weiter fortgefuhrt von v. 32. Der Gedanke in v. 30 ist also derjenige, welcher dem zweiten Liede, Ps. B, zugrunde liegt, wozu dieser Vers den Anfang bildet. Man streiche רִุ in V. 29 nach II S. 22 (Duhm) und nehme יהוה zum ersten Stichos. In v. $29 \mathrm{~b}$

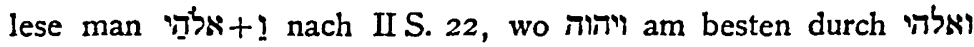
des Psalmes ersetzt wird; LXX A hat in II S. 22 kai kúpıó

v. 31. Der dritte Stichos dieses Verses hat fünf Hebungen statt drei, außerdem hat dieser Vers drei Stichen, während alle anderen entweder zwei oder vier Stichen haben. Der Stichos: „Ein Schild ist er für alle, die sich bei ihm bergen" ist wahrscheinlich, wie Prof. Stade mit gütigst mitteilte, aus zwei Stichen zusammengeflossen. Infolgedessen emendiere ich nach Ps. 17, 7:

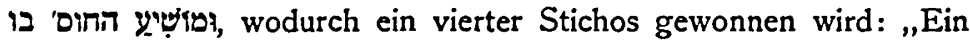
Schild ist er für alle Und ein Erretter derer, die sich in ihm 
bergen". Duhm hegt, wie mir scheint, mit Unrecht Mibtrauen gegen den Vers. Dieser Vers ist, wie auch v. 25 ff., natürlich nicht am Platze in einem Kriegsliede wie Ps. B; die Verse passen aber vorzüglich in dem geistlichen Liede Ps. A, zu welchem sie einen idealen Schluß bilden. Man lese מנן + P.

Psalm B vv. 30. 32-49. 51. 50.

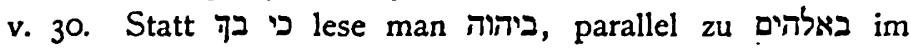
zweiten Stichos, ב instrumenti G.-K. ${ }^{27}$ S I190. Die Verdrängung dieses Verses, verursacht durch das Zusammenschmieden zweier Gedichte, hat vielleicht diesen Schreibfehler verursacht. Der Krieger beginnt sein blutiges Werk mit Hülfe Jahwes. Für die anderen notwendigen Emendationen siehe Lagarde.

v. 32 führt v. 30 weiter fort (Duhm). Statt 7 möchte man

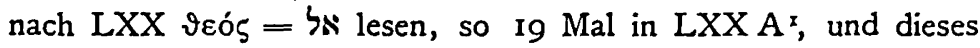
gleich ל $=$ Führer setzen, Hes. 3I, II. In dieser Bedeutung paßt das Wort vorzüglich in den Zusammenhang des Gedichtes, jedoch ist das Wort selbst von unsicherer Existenz. Des Rhythmus halber schreibe man "כי־מי-אל.

v. 33. Der Artikel vor מאוארנ ist grammatisch gut möglich, G.-K. ${ }^{27} § I 16 f$, da er aber bei den anderen Partizipien fehlt v. 34. 35, so ist er vielleicht hier nicht ursprünglich (so Duhm). Nach Baethgen vertritt der Artikel das Relativum.

v. 33f. תמים ist hier nicht von der sittlichen Beschaffenheit gebraucht (Baethgen) wie in v. 26 , sondern im physischen Sinne

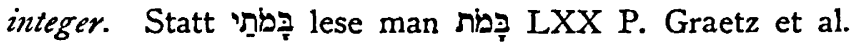

v. 36. Das Versmaß $3+2+2$ stimmt nicht mit dem der an-

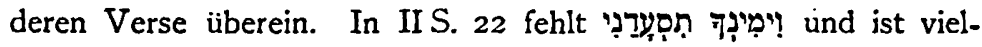
leicht im Ps., wie Duhm bemerkt, ein Ersatz für den unverständ-

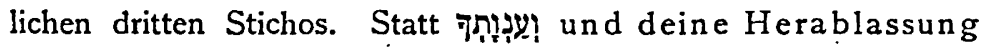

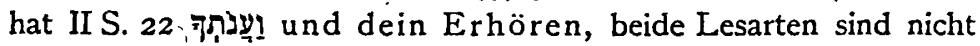

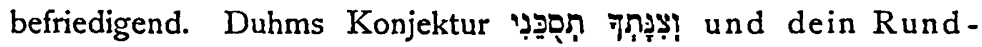
schild beschirmt mich ist rhythmisch zu kurz. Die Lesart

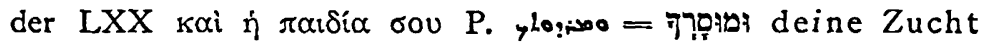
statt וענותך ist vielleicht das Richtige.

I Vgl. IViegand: Der Gottesname צור etc. ZAW 1890 S. 87. 
v. 4I. Des Parallelismus halber liest man besser mit LXX hier und in II S. 22 תצמיתם (Baethgen). Der zweite Stichos hat nur zwei Hebungen.

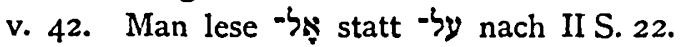

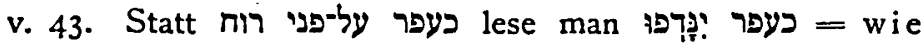
Staub werden sie verweht Ps. 68, 3 und streiche חר, welches vielleicht aus Ps. I, 4 in diese Stelle hinein gelangt ist, des Rhythmus halber. - Man lese nach IIS. 22 fest statt kot aber festgetreten.

v. 44. Das Metrum dieses Verses ist nicht in Ordnung, der Vers hat 3 Stichen, dasselbe ist der Fall in v. 49; man kann entweder v. $49 \mathrm{c}$ zu v. 44 nehmen (Bickell, Duhm) oder v. $44 \mathrm{c} \mathrm{zu}$ v. 49. Das letztere ist vorzuziehen, da in dieser Aussage v. $44 \mathrm{C}$ und v. 49 der Höhepunkt der Macht des „Ich" Ausdruck findet und die Verse $45 \mathrm{ff}$. darauf vorbereiten. - Statt עa עa lese man nach II S. 22 und Cod. U. Die Bezugnahme ist auf das Volk des Sprechers.

v. 46. יבלבי: vergehen gibt keinen guten Sinn in dieser Verbindung. Unterjochte Völker kommen nicht mit leeren Händen, deshalb lese man מִגנוחות יוברילו לִי Geschenke bringen sie mir. ' ist konstruiert mit $ל$ der Person Ps. 68, 30; 76, 12 und dem Akk. der Sache Zeph. 3, 10. בני נכר ist irrtümlich aus v. 45 hierher gelangt. Der zweite Stichos ist nicht ganz in der Ordnung, da er nur.zweihebig ist, vielleicht ist ein Wort ausgefallen. Nach den Ansichten der Apokalyptiker sind die Heiden tributzahlende Vasallen (Bousset op. cit. S. 222), eine Ansicht, der auch in v. 45-46 Ausdruck gegeben wird. Dieses spricht für das späte Alter des Psalmes.

v. 50-5I. Vers 50 stand wohl ursprünglich hinter v. 5 I, welcher Vers die direkte Fortsetzung von v. 49 ist (s. v. 44). Durch diese Verstellung bot sich Gelegenheit, למשיחו auf David zu beziehen, wobei natürlich die ursprüngliche Bezugnahme auf

× Vgl. auch Nestle ZAW 1896 S. 324 . 
Hyrcanus verloren ging. לדוד bis zum Schlub ist Glosse, da sonst der Vers drei Stichen hätte.

v. 50 schliebt das Lied in passender Weise. Der Psalm hatte ursprünglich keinen messianischen Inhalt.

\section{Die Theophanie.}

v. 8. Man schreibe "כ nach II S. 22 des Rhythmus halber.

v. 12. Man streiche 1 ת nach II S. 22 (Löhr-Thenius) als

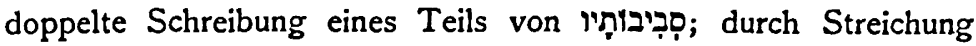
dieses überflüssigen Wortes gewinnt man gleiche Längen für beide parallelen Glieder. Statt i⿱ Die Dunkelheit, die den Gott wie eine Hütte einschliebt, entzieht ihn dem neugierigen Auge des Menschen.

"Dunkelheit des Wassers" kommt nur hier vor, II S. 22, 12 liest משְשרתת"מים ,Menge des Wassers", beide Lesarten passen nicht in den Zusammenhang. Ich schlage vor zu lesen

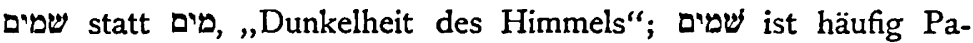

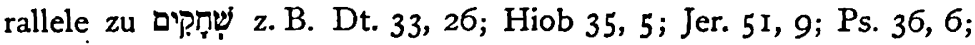
57, II; 108, 5. Gleichfalls muß הو von v. I3 zu v. I2 genommen werden (Bickell, Duhm), um dieses Bild äußerster Dunkelheit zu vervollkommnen. Wir gewinnen durch diese Emendation die nötigen drei Hebungen für diese Zeile.

Der so emendierte Vers ist:

Und er machte Dunkelheit wie eine Hütte um mich herum, Finsternis des Himmels ohne Glanz.

v. I3 ist nicht mit Baethgen "vor ihm verschwanden seine Wolken" zu übersetzen, sondern "vor ihm her zogen seine Wolken", da Jahwe in ihrer Mitte bleibt, Ex. 24, 16f., und die Wolken eine Wand zwischen ihm und den Menschen bilden vgl. v. I2.

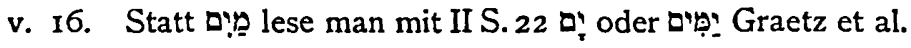

Zum Schlusse möchte ich noch kurz Cheyne's Übersetzung von v. $44-49 \mathrm{c}$ in der Encyclopaedia Biblica III Spalte 395 I erwähnen, welche vielleicht nicht ernst $\mathrm{zu}$ nehmen ist. 
44 a Du befreitest mich von dem Volk der Araber, $49 \mathrm{c} \mathrm{Du}$ errettetest mich von den Leuten von Maaca; 44b Du machtest mich zum Haupte von Nationen, $44 \mathrm{c}$ Völker, die ich nicht kannte, wurden meine Diener; 45 b Die Söhne von Gebal suchten mich ängstlich, 45 a Die Ischmaeliter wurden mir gehorsam; 46 a Sie brachten Weihrauch und Gold, $46 \mathrm{~b}$ Sie boten Ketten von köstlichem Golde dar.

Nach Briggs Studies of the Holy Scriptures S. 412 besteht der Psalm aus vierzehnzeiligen Strophen, nach Duhm aus achtzeiligen. Mir scheint Ps. A ein Gedicht von vier Strophen zu je elf Zeilen $z u$ sein mit einem Einzeiler $z u$ Anfang und zu Ende des Gedichtes, Ps. B hat ebenfalls vier Strophen zu je elf Zeilen. Es besteht aber auch die Möglichkeit, auf die mich Prof. Marti nachträglich aufmerksam gemacht hat, die beiden Psalmen in je 22 Distichen zu zerlegen und sie somit in die Reihe der alphabetisierenden Psalmen aufzunehmen. Jede Zeile des Ps. 18 hatte ursprünglich drei Hebungen, siehe kritische Anmerkungen. 